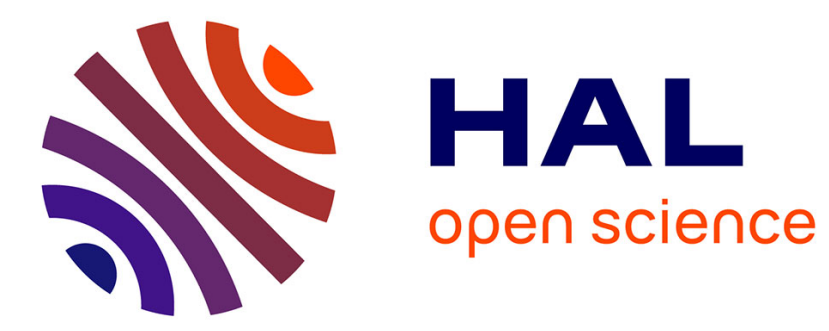

\title{
Les apports de la perspective relationnelle pour comprendre la diffusion d'une politique publique de promotion de la coopération entre PME au Brésil
}

Olivier Coussi, Alsones Balestrin, Kadigia Faccin, Felipe Zarpelon

\section{- To cite this version:}

Olivier Coussi, Alsones Balestrin, Kadigia Faccin, Felipe Zarpelon. Les apports de la perspective relationnelle pour comprendre la diffusion d'une politique publique de promotion de la coopération entre PME au Brésil. Politiques et Management public, 2020, 37 (2), pp.171-192. 10.3166/pmp.37.2020.0009 . hal-02974096

\section{HAL Id: hal-02974096 https://hal.science/hal-02974096}

Submitted on 21 Oct 2020

HAL is a multi-disciplinary open access archive for the deposit and dissemination of scientific research documents, whether they are published or not. The documents may come from teaching and research institutions in France or abroad, or from public or private research centers.
L'archive ouverte pluridisciplinaire HAL, est destinée au dépôt et à la diffusion de documents scientifiques de niveau recherche, publiés ou non, émanant des établissements d'enseignement et de recherche français ou étrangers, des laboratoires publics ou privés. 
Pour citer cet article : Coussi, O., Balestrin, A., Faccin, K., \& Zarpelon, F. (2020). Les apports de la perspective relationnelle pour comprendre la diffusion d'une politique publique de promotion de la coopération entre PME au Brésil. Politiques et Management Public, 37(2), 171-192.

\title{
Les apports de la perspective relationnelle pour
}

\section{comprendre la diffusion d'une politique publique de}

\section{promotion de la coopération entre PME au Brésil}

\author{
Olivier COUSSI • Alsones BALESTRIN ・ Kadigia FACCIN ・ Felipe ZARPELON
}

\section{Résumé}

Le développement de la coopération inter-entreprises est un enjeu majeur des politiques gouvernementales de soutien à la compétitivité économique. Afin d'optimiser la diffusion et l'impact de ces politiques, les pouvoirs publics déploient des dispositifs spécifiques au sein desquels les universités peuvent avoir une place centrale. Si ces dernières ont toute légitimité d'être actives dans le cadre de dispositifs de soutien à l'innovation ou au transfert de technologies, il semble moins naturel de les impliquer pour des opérations en dehors de ces champs. Nous présentons les enseignements issus d'une étude de cas longitudinale sur un programme de coopération inter-entreprises déployée dans un Etat au sud du Brésil par le biais d'un partenariat entre le gouvernement et des universités communautaires en charge de sa mise en œuvre opérationnelle. En nous inscrivant dans une perspective relationnelle, nous analysons les déterminants de ce partenariat afin d'identifier les rentes relationnelles produites par les réseaux de PME construits en véritables écosystèmes territoriaux: l'accès à la communauté et la propagation. Nous démontrons que ces rentes sont confrontées à des obstacles, constituant de fait des pertes relationnelles à minimiser afin d'optimiser l'efficacité de la politique publique poursuivie : l'inflexibilité ainsi que la distance par rapport à la cible.

Mots clés : Perspective relationnelle, université, coopération, étude de cas, Brésil, Politique publique 


\section{INTRODUCTION}

Avec la complexité croissante des relations économiques induites par une participation plus incisive et importante des petites et moyennes entreprises (PME) dans le jeux concurrentiel (Castells, 2009), la question de la capacité des politiques publiques à agir et toucher la totalité de la diversité des acteurs économiques d'un territoire se pose. Constatant cette transformation de configuration des milieux économiques parallèlement à l'évolution des relations entre les sphères publique et privée, les pouvoirs publics doivent, contrairement au passé ou ils traitaient avec un petit groupe de grandes entreprises, impliquer un plus grand nombre d'acteurs aux objectifs plus complexes et parfois dualistes. Paradoxalement, on constate qu'ils sont généralement incapables de faire face efficacement à une grande quantité d'acteurs lors du déploiement de politiques publiques (Klijn, 2008), ce qui nécessite d'identifier des ressources permettant de pallier ce problème.

Les responsables publics peuvent alors impliquer des acteurs non gouvernementaux dans les processus d'élaboration et de diffusion des politiques publiques afin d'atténuer ces problèmes. Ils peuvent choisir d'opter pour la mise en place d'actions collectives dans une approche de type cluster (M. Porter, 1998) avec des avantages comparatifs qui relèvent de la rente ricardienne en économie classique où chaque pays gagne à se spécialiser dans la production où il possède un avantage relatif, souvent par économie d'échelle. En France, cela s'est traduit par la création de différentes politiques publiques telles que les systèmes productifs locaux (SPL) (Courlet, 2000), les pôles de compétitivité (Jacquet \& Darmon, 2005) et en dernier lieu les grappes industrielles (Knecht \& Lacave, 2015).

Assurant la gouvernance d'écosystèmes territoriaux, ces entités ont toutefois souvent souffert de la critique concernant la sous-représentativité des PME en leur sein ${ }^{1,2}$ (Ben Hassine \& Mathieu, 2017; CNEPI, 2017; Lafféter, 2013), au profit des grandes entreprises plus à-même d'un tirer un bénéfice. Enfin, ces politiques sont souvent associées aux objectifs de soutien à

\footnotetext{
1 La Banque des territoires a publié le 18 janvier 2008 sur son site internet un billet (https://www.banquedesterritoires.fr/les-poles-de-competitivite-sont-ils-efficaces) dont on peut extraire le verbatim suivant en réponse à la question «Les PME, victimes d'un manque de considération? : "Outre le surnombre des pôles en France, la faible participation des PME est la critique la plus souvent formulée à leur égard. A y regarder de plus près, c'est davantage d'un manque de valorisation au sein des pôles dont elles souffrent. »

${ }^{2}$ Dans un rapport d'information à la commission de l'économie du Sénat, les rapporteurs Michel Houel et Marc Daunis soulignent les difficultés persistantes spécifiques aux PME ainsi que la nécessaire amélioration de l'intégration des PME comme enjeu majeur (Houel \& Daunis, 2009, p. 34-42)
} 
l'innovation par valorisation de la recherche publique ou bien encore dans une vision très «technologique » de l'utilité de construction d'écosystèmes, ce qui exclue de fait tous les secteurs d'activité qui ne nécessitent pas cette implication (Torre \& Zimmermann, 2015). Suire et Vicente (2015), sur la base de la théorie des réseaux, ont de plus montré la nécessité d'un ciblage "plus chirurgical des liens manquants dans la structuration des réseaux » et la nécessité de soutenir «des tiers-lieux où émergent des rencontres exploratoires, desquelles peuvent naître des collaborations aux frontières des domaines industriels et technologiques ».

Une difficulté subsiste toutefois dans l'évaluation d'une politique de cluster qui renvoi à deux modèles idéal-typiques : un «économiste » et un «gestionnaire» (Gallié et al., 2014). Le premier modèle s'intéresse à un bilan ex-post (mesure de l'impact économique par des approches quantitative et comparative), le deuxième qui cherche à qualifier in itinere des résultats intermédiaires et des relations de causalités de la politique conduite afin éventuellement de la réorienter.

Parmi les partenaires potentiels des entités gouvernementales, les universités ont fait l'objet d'une attention particulière dans la littérature (Etzkowitz \& Leydesdorff, 1995; Hui et al., 2008) car elles ont la caractéristique d'être intégrées dans la société et le plus souvent alignées sur les besoins communautaires. Comme elles représentent naturellement le lieu de production et diffusion de connaissances, elles peuvent de fait alimenter en ressources les entités gouvernementales en matière d'informations techniques et spécialisées. Malgré l'intérêt de la littérature sur les partenariats gouvernement-université, il semble y avoir toutefois une lacune dans la tentative d'analyser l'impact des universités sur le développement et la mise en œuvre des politiques publiques. Ces constatations fondent la problématique qui a guidé cette recherche : comment les entités gouvernementales peuvent-elles utiliser des partenariats avec les universités pour mettre en œuvre, optimiser et amplifier des politiques publiques?

Les relations inter-organisationnelles ont fait l'objet de nombreux débats dans la communauté scientifique en management stratégique, et l'approche théorique que Dyer et Singh (1998) définissent comme la «perspective relationnelle » apporte un point de vue particulier. Ils introduisent la notion de rente relationnelle qui désigne l'idée selon laquelle ce sont les relations que l'entreprise entretient avec ses différentes parties prenantes qui permettent d'expliquer une situation de surperformance: par les synergies qu'elles induisent, les coopérations et les alliances peuvent donc accroître les performances et réduire les coûts et les risques. En ce sens ces rentes s'avèrent stratégiques. Mais qu'en est-il lorsque la construction 
de telles coopérations est à l'initiative des pouvoirs publics ? Est-ce que dans ce contexte les résultats de la perspective relationnelles sont toujours applicables et à quelles conditions ?

Afin de comprendre ce problème, nous présentons les résultats d'une étude de cas longitudinale concernant un programme de coopération entre un gouvernement et des universités communautaires afin d'assure un soutien à la création de coopération entre PME au Brésil.

Nous présentons tout d'abord un cadre conceptuel construit à partir du support théorique de Dyer et Singh (1998). Suite à une description détaillée du cas représentatif étudié, nous exposons les résultats pour enfin conclure sur des recommandations managériales issues d'une extension du cadre conceptuel.

\section{RENTES RELATIONNELLES ET POLITIQUES PUBLIQUES}

Nous proposons dans cette section un cadre conceptuel fondé sur le cadre théorique de la perspective relationnelle et son application dans le cadre d'une politique publique.

\subsection{LA PERSPECTIVE RELATIONNELLE}

Considérant la coopération, et pas uniquement le comportement concurrentiel des acteurs, comme une stratégie pour réussir dans les affaires, Dyer et Singh (1998) discutent des sources d'avantages concurrentiels et se trouvent confrontés à deux grandes lignes de pensée stratégique : la structure des industries caractérise la première voie, et la vision par les ressources internes, en tant que critique à cette première, se positionne en pensée alternative. Porter (1998) est sans aucun doute le principal défenseur du courant de pensée sur la structure des industries en tant que source d'avantage concurrentiel profitable. La deuxième voie, celle basée sur les ressources (Ressource Based-view ou RBV) est issue des travaux précurseurs de Barney (Barney, 1991) et propose que les avantages concurrentiels d'une organisation proviennent de ressources propres. Malgré la reconnaissance de ces deux visions, Dyer et Singh (1998) soutiennent qu'elles négligent les relations de coopération entourant l'organisation de base confirmé en cela par Preston et Donaldson (1999) qui considèrent que ces arrangements constituent des «avantages relationnels » qui devraient être considérés, au même titre que les avantages physiques et financiers. Pour autant, ces derniers constatent qu'en ne prenant en considération que les relations entre entreprises - les relations avec des organisations de nature différentes ne sont pas prises en compte - il existe des lacunes dans cette approche, lacune que nous proposons de combler en proposant d'inclure les relations avec les institutions publiques. 
En effet, la perspective relationnelle perçoit les organisations et institutions environnantes (concurrents, clients, fournisseurs, etc.) en tant que possibilités de collaboration immédiates (Prahalad \& Ramaswamy, 2004) et cherche à comprendre comment et pourquoi les gains relationnels (définis en théorie comme des « rentes relationnelles ») existent et comment ils définissent des avantages concurrentiels pour les organisations participant à des projets collaboratifs. Le modèle de performance dans cette perspective repose alors sur le partenariat qui permet de générer des profits supérieurs. Pour la perspective relationnelle, la stratégie collective crée une source inimitable de ressources grâce à un réseau ayant un accès privilégié à l'information et au partage des connaissances. Pour autant, les rentes relationnelles ne sont pas accessibles en se confrontant directement au marché mais par la constitution de quatre déterminants permettant de les générer (Dyer \& Singh, 1998, p. 663) :

1. Investissements dans des actifs spécifiques à la relation: les organisations en collaboration doivent investir dans la création de ressources uniques qui ne seraient pas développables sans une relation de coopération.

2. Un échange substantiel de connaissances : la connaissance doit être partagée par les routines quotidiennes établies par les partenaires. Elle est conditionnée par la capacité d'absorption spécifique des deux parties ainsi que par les initiatives mises en place favorisant des rapports transparents, sincères, et décourageant tout comportement opportuniste de nature à nuire à la relation entre les parties.

3. La combinaison et complémentarités de ressources et de capacités : "ressources distinctives des partenaires de l'alliance qui génèrent collectivement des rentes plus importantes que la somme de celles obtenues à partir des dotations individuelles de chaque partenaire » (Dyer \& Singh, 1998, p. 666).

4. Des mécanismes de gouvernance efficaces afin de réduire les coûts de transaction.

La reconnaissance des aspects relationnels en tant qu'éléments moteurs de l'avantage concurrentiel a changé les principes traditionnels en stratégie : la collaboration représente donc une ressource stratégique pour atteindre les besoins organisationnels de développement ou de soutenabilité (Persais, 2004). Nous ambitionnons de démontrer que c'est aussi vrai dans la création et la mise en œuvre des politiques publiques.

\subsection{LA PERSPECTIVE RELATIONNELLE DANS LE CONTEXTE DES POLITIQUES PUBLIQUES}

Les révolutions concurrentielles soulignées par Castells (2009) se répercutent également dans la sphère publique qui jusque dans les années 1970 ne devaient s'occuper que d'un petit nombre d'organisations puissantes responsables de la plupart des recettes fiscales et de 
l'emploi de main-d'œuvre. À partir des années 1970, le marché concurrentiel atteint un niveau de complexité plus élevé avec une plus grande participation des petites entreprises et des arrangements collaboratifs. Pour faire face à cette complexité, les pouvoirs publics ont vu le besoin éminent d'adopter des partenariats collaboratifs. Pour Klijn (2008), la formulation et la mise en œuvre de politiques publiques intègrent une profusion de relations interorganisationnelles. Saad, Zawdie et Malairaja (2008) introduisent alors la capacité gouvernementale à traiter avec d'autres entités organisationnelles comme moteur pour obtenir des résultats positifs dans le développement des territoires. Afin de promouvoir l'efficacité des ressources publiques, une entité gouvernementale peut ainsi développer un partenariat avec d'autres entités publiques (Sorrentino et al., 2011) ou même avec des organisations de la société civile (Korsching \& Borich, 1997). Parmi les nombreux partenaires potentiels des pouvoirs publics, les universités occupent une place de choix car elles disposent d'un personnel spécialisé et qualifié pour leur concéder des connaissances techniques et scientifiques. En outre, elles sont intégrées dans la société et s'harmonisent généralement avec les besoins de la communauté. Cela semble donc pouvoir prodiguer aux gouvernements une aptitude à mettre en œuvre et propager efficacement une politique publique.

\subsection{CONSTRUCTION D'UN CADRE CONCEPTUEL}

Le rapprochement entre la perspective relationnelle et le management public donne l'occasion d'élaborer le cadre conceptuel support de la recherche (figure 1). Selon la perspective relationnelle, un groupe de quatre déterminants est nécessaire pour atteindre des rentes relationnelles et nous faisons la proposition ici qu'une relation avec un acteur spécifique, en l'espèce une université, est constitutif pour le gouvernement de création d'une rente relationnelle. En conséquence, les rentes relationnelles produites par l'ensemble des relations avec les universités impliquées dans un programme sont génératrices de succès permettant l'atteinte des objectifs de la politique publique ; a contrario, l'absence de relations avec des universités n'auraient pas produits de rentes relationnelles et donc les objectifs de la politique publique n'auraient pas été atteints conduisant ainsi à son échec.

Les rentes relationnelles sont des supra-bénéfices générés conjointement dans une relation d'échange qui ne pourraient être obtenue par l'une ou l'autre des organisations isolément et ne pourraient être créées que par les contributions conjointes et idiosyncratiques des partenaires spécifiques de l'alliance (Dyer \& Singh, 1998). Dans le contexte de la mise en œuvre des politiques publiques, une rente relationnelle serait donc la capacité à faciliter son application et améliorerait son efficacité (Klijn, 2008). Nonobstant, elle devrait naturellement découler 
d'un objectif commun de développer un secteur spécifique de la société.

En effet, un gouvernement doit être reconnu comme une entité capable de suggérer des changements dans des communautés spécifiques. Au sens large, les groupes sociaux acceptent les pressions extérieures lorsqu'ils reconnaissent la légitimité des acteurs qui en sont partisans (Suddaby \& Greenwood, 2005; Zietsma \& Lawrence, 2010). En raison de ses liens étroits avec la communauté (Hui et al., 2008), l'université peut servir de levier pour permettre l'accès des politiques publiques aux routines quotidiennes de cette même communauté, d'autant plus qu'avec plusieurs acteurs répartis sur le territoire, il est encore plus difficile pour un acteur public d'atteindre chaque acteur spécifique et de garantir la participation démocratique de tous. Une fois de plus, nous postulons que les universités peuvent compléter ce manque de ressources des pouvoirs publics et mettre à disposition leur personnel afin de permettre la propagation d'une politique publique, faciliter des coopérations et jouer - si besoin - un rôle d'interface et/ou de régulateur.

Dans la figure 1, nous mettons en évidence les principaux éléments qui seront pris en considération lors de l'analyse du cas. Les déterminants des rentes relationnelles sont connus de la littérature alors que les rentes relationnelles restent à identifier pour un but à atteindre : l'efficacité de la politique publique qui ne se produit qu'en cas de création de ces rentes.

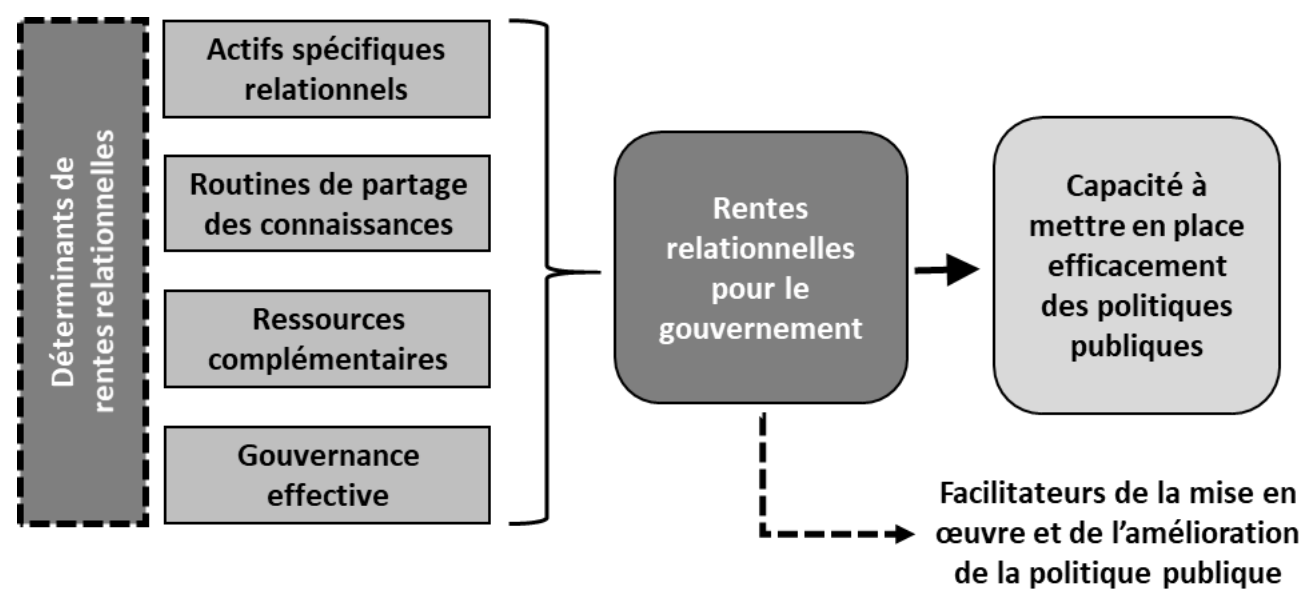

Figure 1. Rentabilité relationnelle et mise en œuvre des politiques publiques (Source: Auteurs adapté de Dyer \& Singh (1998))

\section{LE «PROGRAMA DE COOPERAÇÃO» OU «PROGRAMME DES RESEAUX DE COOPERATION » COMME CAS D'ETUDE}

Le cas étudié décrit une politique publique dans le sud du Brésil qui vise à la formation et à la diffusion de réseaux d'entreprises dans l'Etat du Rio Grande do Sul. Le Rio Grande do Sul est 
un État, unité fédérative intermédiaire, du Brésil. C'est l'État le plus au sud, bordé à l'ouest par l'Argentine et l'Uruguay et à l'est par l'océan Atlantique. Sa population est estimée à 11,2

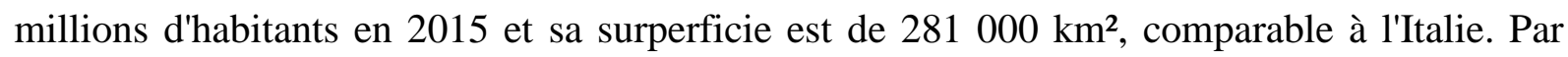
ailleurs, le Rio Grande do Sul est l'un des Etats les plus industrialisés du Brésil avec une base économique très diversifiée et une forte concentration de PME. Compte tenu de ces caractéristiques, le gouvernement de l'Etat a conçu, au début des années 2000, une série de mesures visant à promouvoir le développement économique de l'Etat, en donnant la priorité à la conception de politiques publiques pour la promotion des petites et moyennes entreprises. Parmi ces mesures figure le «Programa de Cooperação» (Programme des Réseaux de Coopération ou PRC), programme visant à «renforcer les micros, petites et moyennes entreprises dans les segments les plus variés de l'économie, par l'union associative entre eux» selon le décret 42.950 de l'Etat du 17 mars 2004. Lancé grâce au partenariat avec une université communautaire et sept consultants, le programme est toujours actif en 2017. En tant que coordonnateur des politiques publiques, le gouvernement de l'Etat a signé des partenariats avec des universités communautaires afin mettre en place une méthodologie d'accompagnement des PME grâce à des actions collaboratives et la constitution de réseaux.

Après une période d'essai, en 2003, la politique publique a été reconfigurée et a étendu les partenariats à sept universités situées aux frontières de l'État. Cette période d'expansion a duré jusqu'en 2007, lorsque la politique publique a souffert d'une diminution des ressources, culminant avec un écart entre 2010 et 2013 sans résultats pertinents. Quoi qu'il en soit, après une nouvelle reconfiguration, la politique publique a repris ses activités en se concentrant sur la gestion et la gouvernance des réseaux de coopération établis. Même si la trajectoire décrit des périodes de rétractation et d'expansion à la politique publique, on peut reconnaître le fait qu'elle a toujours été active tout au long de ses 15 ans d'existence. Même pendant la phase de stagnation, le programme a offert un soutien à un certain nombre de réseaux, que ce soit à l'initiative des universités ou par des techniciens fournis par le gouvernement. Pour ces raisons, ce cas est unique et représentatif en tant que politique publique efficace qui a tenu depuis la première année d'existence le partenariat avec les universités, donnant les moyens d'analyser le rôle des universités dans la mise en œuvre des politiques publiques.

L'étude de ce programme illustre empiriquement l'intérêt de la promotion de la coopération entre entreprises pour le développement local basé sur l'accroissement de la compétitivité des PME. Suite à sa promulgation officielle, le PRC est devenu une référence de politique publique pour la formation et la diffusion de réseaux de coopération entre PME au Brésil. Le 
PRC a été créé avec l'objectif clair de promouvoir une option stratégique pour les petites entreprises avec la formation de réseaux de PME en tant qu'entité légalement établie maintenant l'indépendance et l'individualité de chaque membre. De 2000 à 2015, quatre structures gouvernementales différentes, aux aspirations politiques différentes, étaient responsables de l'organisation du programme et la politique publique est restée active et a atteint des résultats pertinents. Durant son développement, 326 réseaux ont été formés, touchant directement environ 5000 PME, pour une couverture de 45000 emplois et des revenus annuels de 5 milliards de Reals (environ 1,3 milliards d'euros). En raison de la capacité de cette politique publique à rompre avec les pratiques individualistes des PME, il est intéressant de comprendre son fonctionnement tout au long de sa trajectoire. En outre, de nombreuses études récentes présentent les principaux résultats obtenus par les PME participant au programme, parmi lesquelles : l'adoption de nouvelles pratiques de travail, la réduction des coûts, l'acquisition de nouveaux clients, le développement de nouveaux fournisseurs, l'augmentation des revenus et le lancement de nouveaux produits et prestations de service.

En ce qui concerne la dynamique opérationnelle du programme trois acteurs principaux sont identifiés : le gouvernement de l'Etat, des universités communautaires (14 universités ont participé) et des petites et moyennes entreprises. En ce qui concerne les responsabilités et les rôles de chaque acteur, notons que le gouvernement de l'Etat est responsable de la transmission de la méthodologie de travail du PRC (contenant toutes les procédures à suivre par les participants pour le mettre en œuvre), de la fourniture des ressources financières, de la responsabilité de la formation des consultants et de la supervision de toutes les actions liées à la formation de réseaux de coopération. Les universités signataires des accords avec le gouvernement ont créées des centres de soutien régionaux dans tout l'Etat de Rio Grande do Sul qui jouent le rôle stratégique de sensibilisation des entrepreneurs et de constitution des réseaux de coopération, en cherchant à adapter la méthodologie du programme aux potentialités et spécificités de chaque sous-région. Dans les accords signés avec les universités, les critères et les actions à prioriser sont définis, ainsi que les lignes directrices, les objectifs et les phases d'exécution établis par la coordination du gouvernement de l'Etat. La méthodologie développée par le gouvernement pour le PRC suggère une période de 12 mois de travail, divisée en 23 phases, à commencer par la planification de base de l'action et la finalisation avec le plan de croissance et de développement du réseau. En somme, on attend des PME l'adoption de la méthodologie et une appropriation du programme en fonction de 
leurs exigences visant à renforcer leur compétitivité.

Le programme a été créé initialement avec l'objectif clair de promouvoir une option stratégique pour les PME par la formation de réseaux qui maintiennent l'indépendance et l'individualité de chaque membre. Conformément aux nouvelles normes de concurrence (Freeman, 2005), les réseaux d'entreprises sont des relations à long terme, généralement entre PME, qui travaillent ensemble dans le but de maintenir une position concurrentielle face aux concurrents (Jarillo, 1988). Le modèle repose sur l'interconnexion entre les agents de telle sorte que la conception organisationnelle facilite les activités communes et les échanges répétitifs pour garantir un flux continu d'informations, de produits, d'engagements et de confiance (Todeva, 2006).

La formation de ces réseaux repose sur quatre éléments : la coopération horizontale, la nature associative, l'expansion du réseau et l'indépendance vis-à-vis de la sphère publique. Les réseaux de petites entreprises établis dans le sud du Brésil sont des accords de coopération entre PME, entreprises juridiquement indépendantes qui partagent un objectif commun et prennent la décision stratégique de travailler ensemble vers un avantage concurrentiel. Malgré tout, ce sont des projets concurrentiels puisque tous les membres de chaque réseau proviennent d'un même segment partageant - dans certains cas - le même marché. Pour cette raison, ils adoptent comme mode de gouvernance, la coordination par leurs propres membres, élisant des représentants au sein de leurs assemblées.

Enfin, on peut noter que le système d'enseignement supérieur au Brésil est composé d'universités publiques, privées et communautaires, ces dernières étant essentiellement des entités philanthropiques. Les universités publiques sont principalement financées par des ressources fédérales et sont situées dans les grands centres urbains du pays. Bien qu'elles soient indépendantes sur le plan administratif, les universités fédérales sont subordonnées au ministère de l'Éducation et n'ont que peu de souplesse dans la définition des politiques de développement local (Panizzi, 2003). Pour un plus petit nombre, les universités privées font partie d'un phénomène récent de changement du système éducatif brésilien avec l'ouverture d'institutions à but lucratif (Squissardi, 2008). Les universités communautaires (Teixera, 1993) se sont développées historiquement sur des lacunes non comblées par les universités publiques (Souza Lima, 2011). Dans l'Etat du Rio Grande do Sul, les universités ont un rôle prépondérant dans le développement local, souvent dans une approche systémique, écologique et territoriale (Vieira \& Tremblay, 2012) et jouent un rôle particulier dans le développement de l'enseignement supérieur. Ces institutions se développent pour répondre 
aux besoins régionaux non traités par les entités étatiques et fédérales et découlent de l'association des efforts des entités sociales préoccupées par le développement social, économique et culturel des communautés. Ainsi, pour mettre en œuvre cette politique publique, le gouvernement de l'État a choisi les universités communautaires comme opérateurs puisqu'elles sont déjà impliquées dans les différentes communautés du territoire.

\section{METHODOLOGIE DE LA RECHERCHE}

Cette recherche est une étude de cas descriptive et analytique basée sur les procédures méthodologiques de Miles et Huberman (2005), fondée sur une étude de cas unique (Yin, 2012). Le choix de cette méthodologie s'explique par sa visée compréhensive. Nous avons cherché à recueillir la parole des acteurs dans le contexte de leur action, elle permet les « explications pleines de sens »(Yin, 2012). Des trois types d'études de cas distinguée par Stake (1995) nous nous situons dans le cadre de l'étude instrumentale destinée à valider le cadre conceptuel mobilisé.

\subsection{COLLECTE DE DONNEES}

Dans le but de caractériser la trajectoire du PRC et surtout la participation des universités à cette initiative, nous avons recueilli des données à partir de deux sources : secondaire (documents et presse officielle) et primaire (entretiens semi-structurés). L'analyse des documents a été particulièrement importante dans la phase initiale pour aborder le domaine de la recherche. Outre les documents promotionnels sur la politique publique, les rapports de suivi et les textes réglementaires, 67 rapports scientifiques concernant le programme gouvernemental, qu'il s'agisse d'articles, de thèses ou de mémoires, ont constitué la base de l'analyse.

Les entretiens semi-structurés ont permis d'approfondir la compréhension des étapes de la trajectoire du programme et aussi de relations qu'il ne serait pas possible d'extraire de l'analyse des documents. Ainsi, nous avons utilisé les entretiens pour caractériser la participation des universités à la mise en œuvre de la politique publique et aussi les déterminants des rentes relationnelles.

Après l'approche initiale du terrain, nous avons structuré l'instrument de collecte de données avec deux blocs de questions découlant du cadre théorique. Le premier bloc a apporté des questions concernant les quatre éléments pour atteindre les rentes relationnelles selon Dyer et Singh (1998) : les actifs relationnels spécifiques, les routines de partage des connaissances, les ressources complémentaires et l'efficacité de la gouvernance. Nous avons mis l'accent sur chacun des éléments pour extraire des informations précises sur la relation entre le gouvernement et les universités. Le deuxième bloc s'est concentré sur les questions relatives aux rentes relationnelles 
qui permettraient la mise en œuvre d'une politique publique : l'accès à la communauté et la possibilité de diffusion de celle-ci. Les entretiens ont été conduit jusqu'à l'atteinte de la saturation des données.

Comme la politique publique traite de linteraction directe entre trois sphères socio-économiques (gouvernement, université et entreprises), nous avons réparti les entretiens de manière égale entre ces trois dimensions. Au final, l'échantillon est constitué de 12 répondants, quatre pour chaque dimension. Le tableau 1 présente l'ensemble des personnes interrogées et synthétise leur participation à la politique publique.

Tableau 1 - Description des répondants

\begin{tabular}{ccc}
\hline Dimension & Personne interrogée & Position \\
\hline \multirow{2}{*}{ Entreprise } & Firm1 & Entrepreneur et consultant externe \\
& Firm2 & Président du réseau \\
& Firm3 & Entrepreneur associé \\
& Firm4 & Président du réseau \\
\hline \multirow{2}{*}{ Gouvernement } & Gov1 & Coordinateur du programme \\
& Gov2 & Secrétaire directeur \\
& Gov3 & Coordinateur du programme \\
& Gov4 & Secrétaire directeur \\
\hline Université & Univ1 & Superviseur du programme \\
& Univ2 & Consultant et superviseur du programme \\
& Univ3 & Consultant de programme \\
& Univ4 & Superviseur du programme \\
\hline
\end{tabular}

\subsection{ANALYSE DES DONNÉES}

L'ensemble de ces données (primaires comme secondaires) a été étudié par une analyse de contenus (Miles \& Huberman, 1991) en suivant les hypothèses de Bardin (2011), qui traite l'analyse du contenu comme un ensemble de techniques permettant d'analyser les communications au moyen de procédures et d'objectifs systématiques qui décrivent le contenu des messages. Parmi les techniques, Bardin (2011) identifie l'analyse catégorielle comme celle qui se réfère au déroulement du discours en catégories, dont les critères de choix et de délimitation sont guidés par les thèmes d'investigation liés à l'objet de l'analyse. Les données ont été analysées à l'aide du logiciel NVIVO suivant les principes de l'analyse catégorielle (Bardin, 1993) en se référant 
au déroulement du discours en catégories, desquelles les critères de choix et de délimitation sont guidés par les thèmes d'investigation liés à l'objet de l'analyse (Figure 2).

Dans ce sens, nous avons organisé les données primaires et secondaires collectées. Nous avons enregistré les entretiens et les avons transcrits dans des archives textuelles individuelles par chacun des répondants. En outre, nous avons regroupé les données documentaires en articles, législation, médias et documents promotionnels. Nous avons organisé les données en fonction des deux blocs découlant du cadre théorique : les déterminants des rentes relationnels et les rentes relationnels.

Au cours de la collecte de données, de nouvelles informations sur les résultats du partenariat entre le gouvernement et les universités sont apparues comme de nouvelles catégories. Le manque de souplesse dans les corrections de trajectoire et l'absence de contact direct entre le gouvernement et la cible de la politique publique, bien qu'ils ne soient pas qualifiés de rentes relationnelles, ont été décrits comme des résultats pertinents de la relation. Nous avons analysé ces catégories et ajouté au cadre théorique, comme un résultat involontaire des relations dans la mise en œuvre des politiques publiques.

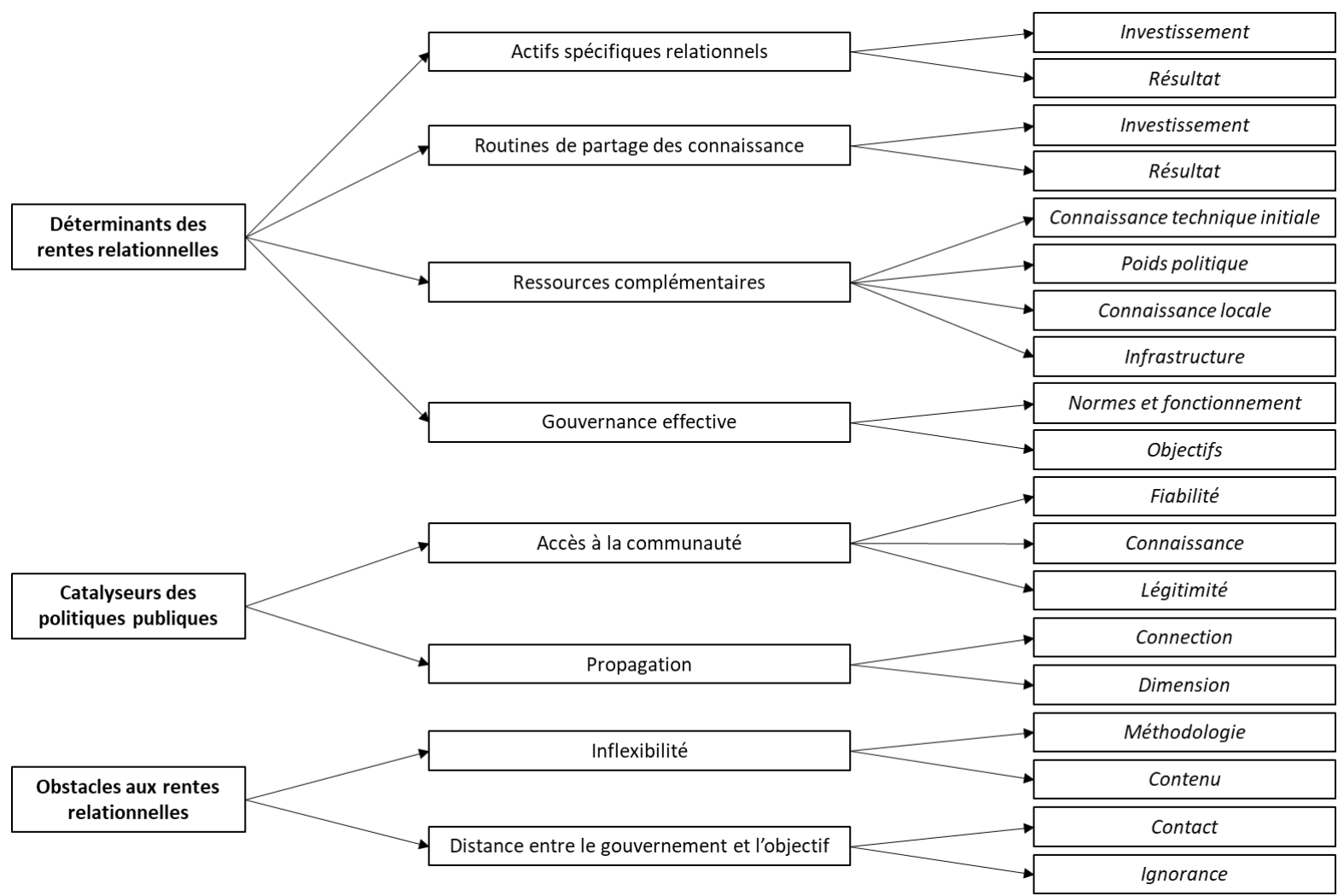

Figure 2. Thèmes d'analyse 


\section{PRINCIPAUX RESULTATS ET CONTRIBUTIONS}

\subsection{DETERMINANTS DES RENTES RELATIONNELLES}

Il s'agit dans un premier temps de démontrer que le cadre théorique proposé s'applique bien au cas étudié. Dyer et Singh (1998, p. 664) soulignent que «Plus l'investissement des partenaires de l'alliance est important dans des actifs spécifiques à la relation, plus le potentiel des rentes relationnelles sera important ». Ainsi, il est remarquable de constater que l'investissement des deux parties du partenariat, gouvernement et universités, se situe uniquement dans un actif spécifique qui serait inaccessible sans participation mutuelle : la reconnaissance de la part des entrepreneurs. En effet, même si l'entité gouvernementale est par nature un acteur légitime dans tout contexte organisationnel, dans les premiers stades de la trajectoire de la politique publique étudiée les entrepreneurs n'ont pas vu dans ces actions une opportunité de développement. Le rôle du gouvernement (Lundberg \& Andresen, 2012), dans la plupart des économies, consiste simplement à déterminer et à contrôler la bonne exécution de textes de lois et de de règlements : «lorsque le programme nous a été présenté, nous avons pensé que ce serait un autre mécanisme gouvernemental de taxation »(Firm1). D'autre part, l'université dans de rares cas possède la structure financière pour appliquer des programmes de soutien au développement d'entreprises environnantes, c'est pour cela que «nous (l'université) avons besoin d'un soutien financier pour fournir notre comité technique comme consultants afin de développer ce type d'initiative» (Univ2). Pour cette raison, l'université n'est généralement pas considérée comme capable de soutenir ce type de programme. L'investissement en reconnaissance comme un actif spécifique relationnel est exposé dans les verbatim du tableau 1. Le rôle du gouvernement fût de fournir un soutien financier aux universités, en contrepartie de quoi, l'université assumait la formation ainsi que le déploiement du comité technique constitué en consultants en organisation. Ce jeu gagnant/gagnant a garanti la reconnaissance de cette politique publique et la confiance en celle-ci de la part des entrepreneurs. 


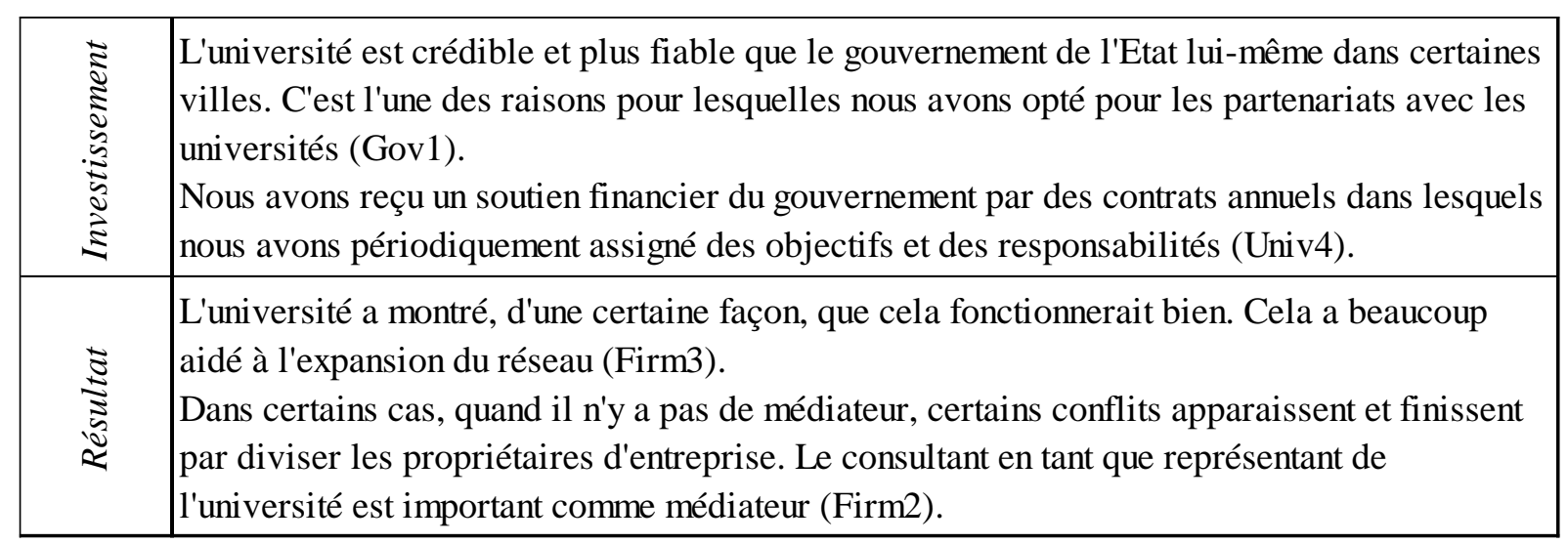

Tableau 1 - Vérification empirique de l'existence d'actifs spécifiques

Le deuxième élément pour générer une rente relationnelle est l'existence de routines de partage des connaissances. Dyer et Singh (1998, p. 665) proposent que «plus l'investissement des partenaires de l'alliance est important dans les routines de partage des connaissances entre entreprises, plus le potentiel des rentes relationnelles sera important »; ce que nous mettons en évidence au travers de ce cas. La méthodologie initiale pour la formulation et le développement de réseaux de coopération entre les PME est la propriété intellectuelle du gouvernement de l'Etat. Les techniciens gouvernementaux avec l'aide d'un consultant externe, président de l'un des premiers réseaux de coopération installés dans l'Etat de Rio Grande do Sul, ont été chargés de formuler cette méthode en 24 étapes. Ensuite, dans les premiers temps de la mise en place de la politique publique, le gouvernement a préparé des séquences immersives avec tous les consultants universitaires dans un hôtel "pour établir une connaissance commune de la méthodologie »(Univ3). Plus tard, le gouvernement a proposé des réunions systématiques pour reconfigurer la méthodologie selon les demandes des consultants. Le programme a ainsi forgé plus de 300 réseaux de coopération au cours de ses 15 années d'existence et il existe un schéma de fonctionnement clair dans tous ces réseaux. En outre, et comme effet collatéral, la plupart des connaissances développées dans les limites de la politique publique sont maintenant intégrées par les groupes de consultants externes et dans le corps professoral impliqué. Les efforts visant à investir dans des routines de partage des connaissances sont notées dans le tableau 2. 


\begin{tabular}{|c|l|}
\hline \multirow{2}{*}{} & Chaque fois que nous avions une nouvelle équipe de consultants, nous avons promu un \\
événement sur deux jours entiers, généralement organisés dans un hôtel, où nous avons passé \\
au crible chaque étape de la méthodologie (Gov3).
\end{tabular}

Tableau 2 - Vérification empirique de l'existence de routine de partage de connaissance

Le troisième facteur déterminant des loyers relationnels est la dotation de ressources complémentaires. Du côté du gouvernement, ce sont les connaissances techniques initiales et le pouvoir législatif. Ces deux ressources étaient essentielles pour initier le processus et pour maintenir la légitimité de la politique publique. Pour compléter ces ressources, les universités ont apporté leurs connaissances des communautés locales et leur capacité d'accès aux entrepreneurs (tableau 3).

\begin{tabular}{|c|c|}
\hline 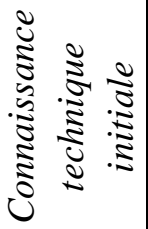 & $\begin{array}{l}\text { Nous avons d'abord développé la méthodologie avec l'aide du président d'un réseau de } \\
\text { coopération consolidé (Gov4). } \\
\text { Elle (le consultant externe) avait en tête toutes les étapes de construction d'un réseau de } \\
\text { coopération. Je devais simplement le mettre sur le papier (Gov1). }\end{array}$ \\
\hline 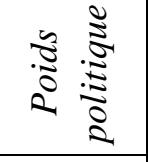 & $\begin{array}{l}\text { Le gouvernement a appuyé la création de deux lois importantes pour les réseaux de } \\
\text { coopération. La première a mis en œuvre le programme et l'autre a facilité la régularisation } \\
\text { des réseaux de coopération en tant qu'associations économiques (Univ3). }\end{array}$ \\
\hline 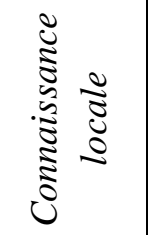 & $\begin{array}{l}\text { L'université connaît le marché et est responsable des prospections (Firm2). } \\
\text { Nous avonsle plus souvent mis en place le comité technique avec une connaissance suffisante } \\
\text { des paramètres économiques locaux (Univ4). }\end{array}$ \\
\hline 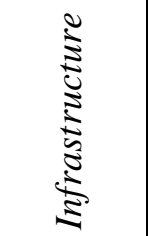 & $\begin{array}{l}\text { Une autre responsabilité de l'université était de fournir des salles de classe et du matériel, } \\
\text { tels que des manuels et des livres techniques, pour former les entrepreneurs (Univ1). }\end{array}$ \\
\hline
\end{tabular}

Tableau 3 - Vérification empirique de l'existence de ressources complémentaires

Concernant l'efficacité de la gouvernance, notons que les règles de coordination dans toute relation sont sources de diminution des coûts de transaction. De cette façon, Dyer \& Singh (1998) proposent que la capacité de minimiser les coûts de transaction et de maximiser la valeur soient de véritables facteurs de rentes relationnelles. Les règles de coordination dans le 
cas étudié ont été établies par l'entité gouvernementale (tableau 4).

\begin{tabular}{|c|c|}
\hline 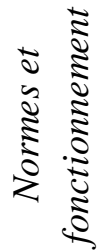 & $\begin{array}{l}\text { Nous (les universités) avons été responsables de la fourniture de consultants et } \\
\text { d'infrastructures (Univ4). } \\
\text { Le gouvernement a été responsable du soutien financier et de la formation sur la } \\
\text { méthodologie (Gov1) }\end{array}$ \\
\hline $\begin{array}{l}\frac{n}{0} \\
\frac{0}{0} \\
\frac{0}{0}\end{array}$ & $\begin{array}{l}\text { Chaque université devait développer un certain nombre de réseaux de coopération en } \\
\text { fonction de ses besoins locaux (Univ1). } \\
\text { En tant que consultants, nous avons eu pour objectif de créer un certain nombre de réseaux de } \\
\text { coopération et un objectif de soutien à un certain nombre de réseaux de coopération déjà } \\
\text { établis (Univ2) }\end{array}$ \\
\hline
\end{tabular}

Tableau 4 - Vérification empirique de l'existence d'une gouvernance effective

Dès que le gouvernement s'est rendu compte de son incapacité à mettre pleinement en œuvre la politique publique avec ses propres ressources, le coordinateur du programme a établi plusieurs règles pour réaliser le partenariat avec les universités. Cet ensemble de règles a été discuté avec la première université impliquée dans le programme et, après des ajustements exigeants, il y eut contractualisation. Comme l'explique le superviseur du programme, «le contrat, renouvelable annuellement, établissait les objectifs et la responsabilité des deux parties»(Univ1). Une fois le contrat mis en place, l'entité gouvernementale a établi des mécanismes de gouvernance: la coordination du programme incombe au secrétaire du développement économique (entité gouvernementale) et chaque université dispose d'un superviseur qui est responsable du déploiement des objectifs de chaque consultant. Chaque mois, le superviseur fournit un rapport concernant le nombre d'entreprises visitées, des détails sur les entreprises (par exemple, le nombre d'employés, les revenus annuels, le secteur économique) et également des indicateurs concernant l'efficacité des consultants (par exemple, le taux d'adhésion et de décrochage, et l'évolution du groupe à travers les 23 étapes de la méthodologie). Comme on le voit, les quatre éléments déterminants de production de rentes relationnelles sont explicites, correspondent aux propositions théoriques et sont constitutifs d'une alliance collaborative fructueuse.

\subsection{LES RENTES RELATIONNELLES A LA MISE EN CEURE DE LA POLITIQUE PUBLIQUE}

Comme nous l'avons déjà exposé, le gouvernement de l'Etat du Rio Grande do Sul n'aurait pas la capacité de communiquer pleinement avec tous les entrepreneurs directement concernés par la politique publique qu'il souhaite mettre en œuvre : d'une part il n'est pas capable de fournir un soutien organisationnel technique et d'autre part, les universités peuvent être légitime pour 
faciliter l'accès du gouvernement à la communauté. Lors du premier accès à la communauté, dans les premiers instants de la politique publique, l'apport des universités était essentiel. Le premier coordinateur du programme affirme ainsi que «nous (le gouvernement) ne pouvions pas rendre cela possible sans que les universités nous aident»(Gov1) : cela est constitutif d'une rente relationnelle, car le travail isolé des deux parties n'atteindrait pas le même résultat. La seconde rente relationnelle se rapporte à la capacité du gouvernement à diffuser la politique publique auprès d'une grande quantité d'individus. Selon la littérature, les partenariats entre les entités gouvernementales peuvent renforcer le pouvoir politique pour mettre en œuvre les politiques publiques (Korsching \& Borich, 1997; Sorrentino et al., 2011). Quoi qu'il en soit, dans ce cas, l'entité gouvernementale a cherché dans le partenariat une capacité de levier au sein de l'ensemble de son territoire dont la superficie est proche de celle de l'Italie. Malgré cette dimension importante, le secrétaire du développement économique ne disposait que de quatre techniciens pour ce programme, d'où l'impossibilité d'atteindre tous les territoires de l'Etat sans aide extérieure. Les universités ont alors utilisé, dans la plupart des cas, leurs propres étudiants comme consultants pour mettre en œuvre les pratiques de création d'un réseau. De cette façon, le gouvernement a pu agir dans toutes les régions de Rio Grande do Sul et diffuser le contenu de la politique publique. Il est ainsi clair que le gouvernement, en travaillant seul, n'aurait pas atteint les objectifs du programme. Ces deux rentes relationnelles soulignent l'effet de levier des universités dans la mise en œuvre des politiques publiques comme en témoigne les verbatim du tableau 5. 


\begin{tabular}{|c|c|c|}
\hline \multirow{3}{*}{ 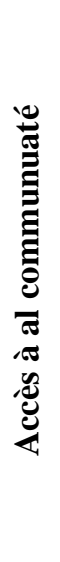 } & 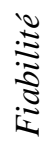 & $\begin{array}{l}\text { Dans certains contextes, les pouvoirs des universités sont vraiment fiables (Gov1). } \\
\text { Lorsque nous (consultants) sommes en face des entrepreneurs et qu'on montre une carte de } \\
\text { visite de l'université, nous constatons la confiance qu'ils avaient en nous (Univ2). }\end{array}$ \\
\hline & 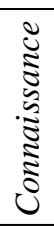 & $\begin{array}{l}\text { Dans certains cas, lorsqu'il n'y a pas de médiateur, certains conflits apparaissent et finissent } \\
\text { par diviser les propriétaires d'entreprise. Le consultant en tant que représentant de } \\
\text { l'université est important en tant que médiateur (Firm2). } \\
\text { L'université est insérée dans la communauté et a cette vision politique d'aider les } \\
\text { entrepreneurs et cela a joué un effet de levir à l'uinitiation du programme (Univ3). }\end{array}$ \\
\hline & 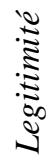 & $\begin{array}{l}\text { L'université était capable d'assembler le réseau parce qu'elle en avait l'autorité (Firm1). } \\
\text { L'université était une autorité qui apportait quelquechose d'officiel et de sécurisé (Firm3). }\end{array}$ \\
\hline \multirow{2}{*}{ 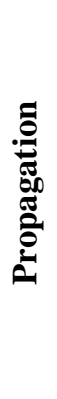 } & 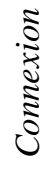 & $\begin{array}{l}\text { Les universités ont dû travailler à l'échelle régionale, puis elles ont fini par embaucher leurs } \\
\text { propres étudiants diplômés en tant que consultants (Gov3). }\end{array}$ \\
\hline & 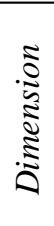 & $\begin{array}{l}\text { (...) en raison des dimensions de l'État, il était important que le gouvernement soit présent } \\
\text { dans certaines régions par le biais de partenaires locaux. L'option a été faite pour les } \\
\text { universités (Gov4). } \\
\text { Nous avons conclu des contrats avec les universités dans le but d'embaucher des personnes } \\
\text { qui souhaiteraient coopérer avec toutes les régions de l'État (Gov1). }\end{array}$ \\
\hline
\end{tabular}

Tableau 5 - Vérification empirique de l'existence de rentes relationnelles

\subsection{OBSTACLES DECOULANT DU PARTENARIAT}

Même si tous les éléments garantissant des rentes relationnelles permanentes sont présents, le dispositif a dû faire face à des obstacles. Nous observons alors des arbitrages dans ce partenariat et nous discutons de leurs implications. Dyer et Singh (1998) négligent en effet les effets secondaires de la collaboration comme moyens de diminuer l'avantage concurrentiel et cette création d'obstacles pour la mise en œuvre effective de la stratégie. Les données empiriques de cette étude de cas suggèrent que les partenariats entre le gouvernement et l'université ont produit au moins deux obstacles à la mise en œuvre effective des politiques publiques.

La première barrière concerne l'inflexibilité de l'entité gouvernementale à changer l'orientation de la politique publique. Il se dégage un consensus pour les trois types d'acteurs impliquées dans cette recherche: le gouvernement n'a pas été en mesure de corriger rapidement la trajectoire du programme. En conséquence, le programme a pris trop de temps pour se réinventer et commencer à proposer une méthodologie différente à celle qui était déjà établie. De 2010 à 2013, le programme n'a pas été en mesure d'avoir des résultats effectifs. Cette inflexibilité provient ainsi de l'incapacité du gouvernement à comprendre le besoin de correction et aussi l'incapacité de mobiliser l'ensemble du réseau pour convenir de 
changements conjoints le cas échéant. De plus, bien que les mécanismes de gouvernance prévoyaient des réunions systématiques pour discuter de l'avenir du programme, "il était presque impossible de parvenir à un accord avec tous les superviseurs "(Univ1). L'inflexibilité comme obstacle à la mise en œuvre de la politique publique est mise en évidence dans le tableau 6.

La seconde barrière strictement liée à l'inflexibilité est la distance entre le gouvernement et l'objectif de la politique publique. En raison de l'incapacité d'atteindre tous les entrepreneurs, le gouvernement a cédé la responsabilité de traiter avec le public auprès des universités. Cela a amené, à certaines occasions, le sentiment de mépris des entrepreneurs : "nous (les propriétaires d'entreprises) ne voyions parfois la volonté de faire que de la part des consultants et pas dans le gouvernement»(Firm1). Cette distance a également causé l'incapacité d'établir une communication entre le gouvernement et les entrepreneurs sans médiateur. De cette façon, l'entité gouvernementale a souvent ignoré les besoins des entrepreneurs car il n'en avait tout simplement pas connaissance : "le flux d'informations a eu des problèmes évidents dans le programme » (Univ4). Le tableau 6 propose d'autres verbatim confortant cette observation.

\begin{tabular}{|c|c|c|}
\hline \multirow{2}{*}{ 曽 } & 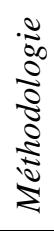 & $\begin{array}{l}\text { Lorsque nous avons terminé la mise en œuvre de la méthodologie de la formulation du } \\
\text { réseau, nous devrions avoir une méthodologie stricte pour suivre le développement de ces } \\
\text { mêmes réseaux. Cela ne s'est produit que récemment en } 2013 \text { (Univ2). } \\
\text { La politique publique était incapable de traiter certaines choses importantes, comme l'arrêt } \\
\text { du soutien aux réseaux. C'est peut-être le plus grand problème (Univ4). }\end{array}$ \\
\hline & 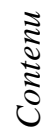 & $\begin{array}{l}\text { Les thèmes tel que confiance, motivations ou motifs d'annumlation du partenariat étaient } \\
\text { importants mais non traités dans le programme (Firm2). }\end{array}$ \\
\hline \multirow{2}{*}{ 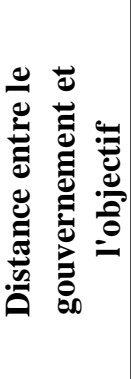 } & 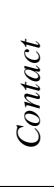 & $\begin{array}{l}\text { La seule connexionfût celle avec les consultants. Il n'y avait pas de contact direct entre le } \\
\text { gouvernement et les entrepreneurs (Firm4). } \\
\text { Nous avons toujours eu du mal à apporter des changements au programme, mais personne ne } \\
\text { nous a entendu (Firm2) }\end{array}$ \\
\hline & $\begin{array}{l}0 \\
0 \\
\vdots \\
0 \\
\vdots \\
0 \\
-0\end{array}$ & $\begin{array}{l}\text { Autant que je sache, le programme provenait de l'université. C'est elle qui est venue me } \\
\text { parler (Firm3). } \\
\text { Nous avons rarement vu un représentant du gouvernement lors des rencontres avec nous } \\
\text { (Firm4). }\end{array}$ \\
\hline
\end{tabular}

Tableau 6 - Vérification empirique de l'existence d'obstacles aux rentes relationnelles

\section{QUELQUES ENSEIGNEMENTS THEORIQUES ET MANAGERIAUX EN GUISE DE CONCLUSION}

Malgré tous les efforts visant à investir dans des actifs spécifiques à la relation, à établir des 
routines de partage des connaissances, à développer des ressources complémentaires et à promouvoir une gouvernance efficace, le partenariat n'a pas pu éviter ce que nous qualifions de pertes relationnelles. Nous proposons qu'un arbitrage est nécessaire au sein d'un partenariat entre l'université et le gouvernement pour mettre en œuvre une politique publique économique.

Ainsi, une fois constaté que les éléments proposés par Dyer \& Singh (1998) ne garantissent pas que seules des rentes relationnelles découlent du partenariat, nous proposons de changer le terme « déterminants des rentes relationnelles » en «déterminants des partenariats ». Nous supposons alors que ces éléments apportent non seulement des rentes relationnelles, mais aussi des pertes relationnelles à un partenariat. L'inflexibilité et la distance à l'objectif de la politique publique au lieu de fonctionner comme des leviers pour mettre en œuvre des politiques publiques, constituent des obstacles qui détournent le gouvernement dans l'atteinte de ses objectifs. Lors de la mise en œuvre d'une politique publique, le manager public devra alors concentrer ses efforts à maximiser les rentes relationnelles tout en prenant garde de minimiser l'impact des obstacles qui existent dans l'exécution de cette politique, obstacles inhérents à la nature même du type de politique poursuivie, a fortiori lorsque celle-ci nécessite la médiation d'un tiers tel qu'une université. Ces observations empiriques conduisent à proposer un nouveau modèle présenté figure 2.

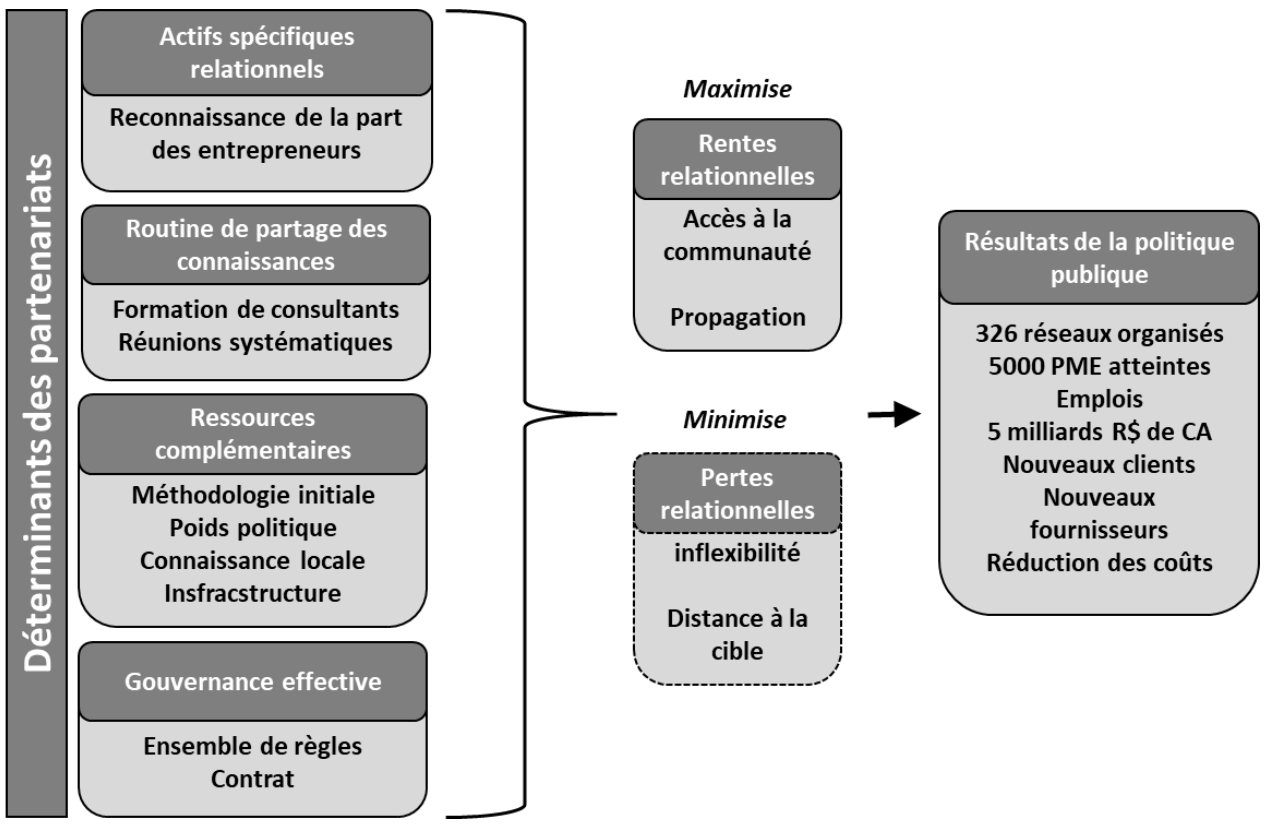

Figure 2. Modèle final

Nous induisions au début de cet article que la construction d'un partenariat avec une université pouvait produire un effet de levier stratégique dans la mise en œuvre d'une 
politique publique de coopération entre entreprises. Le cas du Programme Réseaux de Cooperation, dans l'État de Rio Grande do Sul au Brésil, se révèle riche d'enseignements. En effet, en formalisant un partenariat avec plusieurs universités communautaires, l'Etat de Rio Grande do Sul a pu soutenir la création de plus de 300 réseaux de petites entreprises dans son territoire démontrant par l'occasion tout à la fois la pertinence de la politique publique poursuivie mais aussi sa mise en œuvre opérationnelle et fructueuse. Le gouvernement et les universités ont ainsi investi dans la légitimité comme un atout relationnel spécifique afin de faciliter la communication vers les entreprises concernées par le programme et cibles de la politique publique. En outre, les routines de partage des connaissances mises en œuvre par les deux parties ont permis l'existence de connaissances mutuelles sur la méthodologie et ainsi créer des réseaux de coopération répartis autour de plus de 100 consultants. Ressources complémentaires, en soi, les connaissances techniques initiales, le pouvoir politique du gouvernement et des connaissances locales ainsi que les moyens des universités ont pu créer une forte synergie pour mettre en œuvre la politique publique. Enfin, des mécanismes de gouvernance efficaces dans le cadre d'un contrat ont été établis pour régir le partenariat.

Cette recherche apporte une recommandation claire aux managers publics qui cherchent à construire des accords de coopération pour mettre en œuvre leurs politiques : il faut trouver le parfait équilibre entre la capacité d'action et l'éloignement de la communauté. Même si la coopération peut garantir l'accès et la capacité technique au service de la communauté au sens large, le fait que le pouvoir publique (en ce cas l'État fédéré) ne soit pas le maître d'œuvre de la politique publique, rend le programme moins flexible et parfois incapable de répondre aux demandes réelles de la communauté. Le développement incisif des déterminants des rentes relationnelles (actifs spécifiques, partage des connaissances, interdépendance des ressources et gouvernance) est un moyen de trouver l'équilibre optimal entre capacité d'action et éloignement de la communauté.

Nous contribuons ainsi à la littérature $i$ ) sur la perspective relationnelle car nous offrons une vision complète du partenariat et ii) sur les leviers stratégiques en éclairant le rôle important des universités pour l'appui à la mise en œuvre de politiques publiques. Nous offrons également des contributions aux praticiens, en particulier ceux qui s'intéressent à la mise en œuvre de la politique publique, car nous décrivons les meilleures pratiques pour concevoir un partenariat entre acteur public et université. 


\section{REFERENCES BIBLIOGRAPHIQUES}

Bardin, L. (1993). L'analyse de contenu. Presses Universitaire de France.

Barney, J. (1991). Firm Resources and Sustained Competitive Advantage. Journal of Management, 17(1), 99-120.

Ben Hassine, H., \& Mathieu, C. (2017). Évaluation de la politique des pôles de compétitivité: La fin d'une malédiction? (Document de travail No 2017-03; p. 82). France Stratégie.

Castells, M. (2009). The rise of the network society (Wiley-Blackwell).

CNEPI. (2017). Avis sur la politique des pôles de compétitivité (Commission Nationale d'évaluation des politiques d'innovation, p. 28). France Stratégie.

Courlet, C. (2000). Districts industriels et systèmes productifs localisés (SPL) en France [Rapport final]. DATAR.

Dyer, J. H., \& Singh, H. (1998). The Relational View : Cooperative Strategy and Sources of Interorganizational Competitive Advantage. The Academy of Management Review, 23(4), 660.

Etzkowitz, H., \& Leydesdorff, L. A. (1995). The triple helix-university-industry-government relations: A laboratory for knowledge-based economic development. European Association Study Science and Technology Review, 14(1), 14-19.

Gallié, E.-P., Glaser, A., \& Pallez, F. (2014). Une analyse comparative des évaluations de politiques de clusters en Europe: Vers deux modèles ideal-typiques. Revue Politiques et Management Public, 31(1), 87-111.

Houel, M., \& Daunis, M. (2009). Les pôles de compétitivité: Bilan et perspectives d'une politique industrielle et d'aménagement du territoire (Rapport d'information du Sénat fait au nom de la commission de l'économie n²0 (2009-2010)).

Hui, P. P., Fonstad, N. O., \& Beath, C. M. (2008). Technology service inter-organizational relationships. In The Oxford handbook of inter-organizational relations (Oxford University Press Inc., p. 256-280). Cooper S. et al.

Jacquet, N., \& Darmon, D. (2005). Les Pôles de compétitivité : Le modèle français. (La Documentation française). http://www.ladocumentationfrancaise.fr/ouvrages/3303331952251les-poles-de-competitivite\#book_sommaire

Klijn, E. (2008). Policy and Implementation Networks. Inter-organizational relationships, Chain and Networks : A Supply perspective. In The Oxford Hanbook of Inter-organizational Relations. (Oxford University Press). Steve Cropper, Chris Huxham, Mark Ebers, and Peter Smith Ring.

Knecht, F., \& Lacave, M. (2015). Évaluation de la politique des grappes d'entreprises. CGET.

Korsching, P. F., \& Borich, T. O. (1997). Facilitating Cluster Communities : Lessons from the Iowa Experience. Community Development Journal, 32(4), 342-353.

Lafféter, Q. (2013). Quel bilan pour les pôles de compétitivité français? Idées économiques et sociales, 2(172), 34-44.

Lundberg, H., \& Andresen, E. (2012). Cooperation among companies, universities and local government in a Swedish context. Industrial Marketing Management, 41(3), 429-437.

Miles, M. B., \& Huberman, A. M. (1991). Analyse des données qualitatives: Recueil de nouvelles méthodes. (Traduction de Analysing qualitative data: A source book for new 
methods, 1979, 1984. Beverly Hills : Sage). De Boeck.

Oliver, A. L., \& Ebers, M. (1998). Networking Network Studies : An Analysis of Conceptual Configurations in the Study of Inter-organizational Relationships. Organization Studies, 19(4), 549-583.

Panizzi, W. M. (2003). L'université publique : Une référence pour l'enseignement supérieur brésilien. Politiques et gestion de l'enseignement supérieur, 15(1), 49-64.

Porter, M. (1998). Clusters and the new economics of competition. Harvard Business Review, 76(6), 77-91.

Porter, M. E. (1998). Competitive strategy: Techniques for analyzing industries and competitors: with a new introduction (1st Free Press ed). Free Press.

Prahalad, C. K., \& Ramaswamy, V. (2004). The future of competition: Co-creating unique value with customers. Harvard Business School Pub.

Preston, L. E., \& Donaldson, T. (1999). Stakeholder Management and Organizational Wealth. Academy of Management Review, 24(4), 619-620.

Saad, M., Zawdie, G., \& Malairaja, C. (2008). The triple helix strategy for universities in developping countries: The experience in Malyasia and Algeria. Science and Public Policy, 35(6), 431-443.

Sorrentino, M., Spano, A., \& Bello, B. (2011). Evaluating Local Partnership Incentive Policies : A Realist Approach. International Journal of Electronic Government Research, 7(4), 19-34.

Souza Lima, K. R. (2011). O Banco Mundial e a educação superior brasileira na primeira década do novo século. Revista Katálysis, 14(1), 84-94.

Squissardi, V. (2008). Modelo de expansão da educação superior no Brasil : Predomínio privado/mercantil e desafios para a regulação e a formação universitária. Educação \& Sociedade, 29(105), 991-1022.

Stake, R. E. (1995). The art of case study research. Sage Publications.

Suddaby, R., \& Greenwood, R. (2005). Rhetorical Strategies of Legitimacy. Administrative Science Quarterly, 50(1), 35-67.

Suire, R., \& Vicente, J. (2015). Récents enseignements de la théorie des réseaux en faveur de la politique et du management des clusters. Revue d'économie industrielle, 152, 91-119.

Teixera, G. M. (1993). Les universités «communautaires »-Une expérience brésilienne. Nouveaux cahiers sur l'enseignement supérieur UNESCO, 10.

Torre, A., \& Zimmermann, J.-B. (2015). Des clusters aux écosystèmes industriels locaux. Revue d'économie industrielle, 152, 13-38.

Vieira, P. F., \& Tremblay, G. (Éds.). (2012). Le rôle de l'université dans le développement local : Expériences brésiliennes et québécoises. Presses de l'Université du Québec.

Yin, R. K. (2012). Applications of Case Study Research. Sage.

Zietsma, C., \& Lawrence, T. B. (2010). Institutional Work in the Transformation of an Organizational Field: The Interplay of Boundary Work and Practice Work. Administrative Science Quarterly, 55(2), 189-221. 